\title{
Dispersion retrieval from multi-level ultra-deep reactive-ion-etched microstructures for terahertz slow-wave circuits
}

Chan-Wook Baik, Ho Young Ahn, Yongsung Kim, Jooho Lee, Seogwoo Hong, Jun Hee Choi, Sunil Kim, Sang Hun Lee, So Yeon Jun, SeGi Yu, R. Lawrence Ives, Jong Min Kim, and Sungwoo Hwang

Citation: Appl. Phys. Lett. 104, 021118 (2014); doi: 10.1063/1.4862324

View online: https://doi.org/10.1063/1.4862324

View Table of Contents: http://aip.scitation.org/toc/apl/104/2

Published by the American Institute of Physics

\section{Articles you may be interested in}

Experimental observation of sub-terahertz backward-wave amplification in a multi-level microfabricated slowwave circuit

Applied Physics Letters 107, 191109 (2015); 10.1063/1.4935611

Structural degradation mechanism of multiwalled carbon nanotubes in electrically treated field emission

Applied Physics Letters 96, 023105 (2010); 10.1063/1.3291108

Frequency shifts in two-level ultra-deep reactive ion etched slow-wave structures for $0.1 \mathrm{THz}$ backward-wave oscillations

Applied Physics Letters 101, 073508 (2012); 10.1063/1.4745774

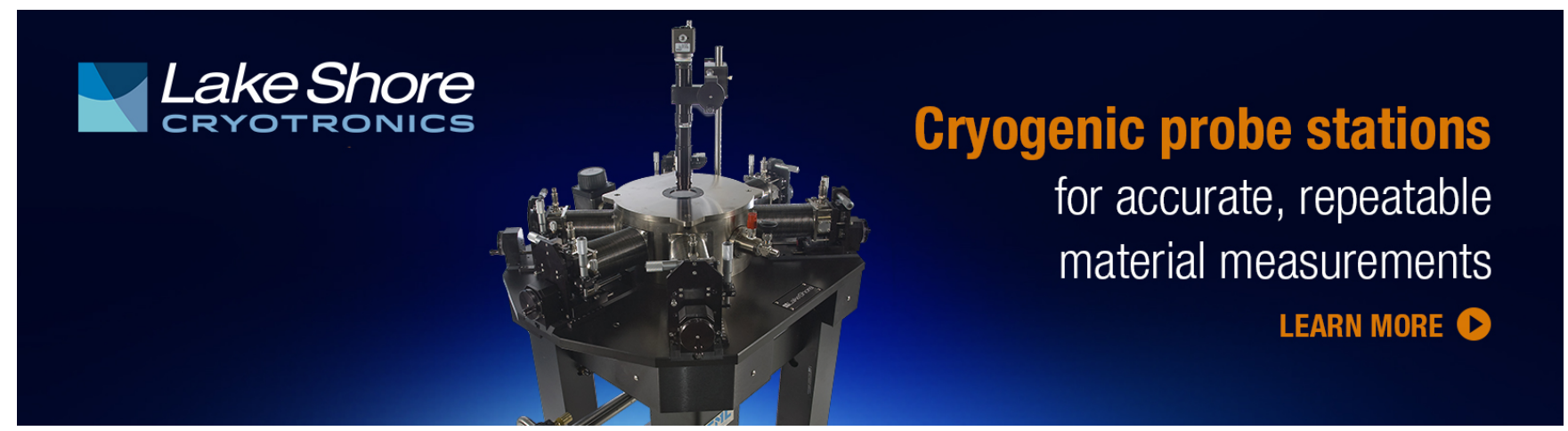




\title{
Dispersion retrieval from multi-level ultra-deep reactive-ion-etched microstructures for terahertz slow-wave circuits
}

\author{
Chan-Wook Baik, ${ }^{1, a)}$ Ho Young Ahn, ${ }^{1}$ Yongsung Kim, ${ }^{1}$ Jooho Lee, ${ }^{1}$ Seogwoo Hong, ${ }^{1}$ \\ Jun Hee Choi, ${ }^{1}$ Sunil Kim, ${ }^{1}$ Sang Hun Lee, ${ }^{1}$ So Yeon Jun, ${ }^{2}$ SeGi Yu, ${ }^{2}$ R. Lawrence Ives, ${ }^{3}$ \\ Jong Min Kim, ${ }^{1, b)}$ and Sungwoo Hwang ${ }^{1}$ \\ ${ }^{1}$ Samsung Advanced Institute of Technology, Yongin 446-712, South Korea \\ ${ }^{2}$ Department of Physics, Hankuk University of Foreign Studies, Yongin 449-791, South Korea \\ ${ }^{3}$ Calabazas Creek Research, Inc., San Mateo, California 94404-1010, USA
}

(Received 5 October 2013; accepted 2 January 2014; published online 16 January 2014)

\begin{abstract}
A multi-level microstructure is proposed for terahertz slow-wave circuits, with dispersion relation retrieved by scattering parameter measurements. The measured return loss shows strong resonances above the cutoff with negligible phase shifts compared with finite element analysis. Splitting the circuit into multi levels enables a low aspect ratio configuration that alleviates the loading effect of deep-reactive-ion etching on silicon wafers. This makes it easier to achieve flat-etched bottom and smooth sidewall profiles. The dispersion retrieved from the measurement, therefore, corresponds well to the theoretical estimation. The result provides a straightforward way to the precise determination of dispersions in terahertz vacuum electronics. (C) 2014 AIP Publishing LLC. [http://dx.doi.org/10.1063/1.4862324]
\end{abstract}

Challenges for generating coherent electromagnetic radiation in terahertz $(\mathrm{THz})$ regime have rapidly arisen due to great interest and potential in advanced physics research and commercial applications. ${ }^{1} \mathrm{THz}$ generation, including detection and manipulation, is currently beyond the capability of most traditional technologies. Microfabricated vacuum electronic devices ( $\mu$ VEDs), however, are promising candidates for versatile, compact, high power, frequency-agile, coherent radiation sources. These micron-precision, lithographic, fabrication methods provide a new path towards millimeter or sub-millimeter wave radiation sources. ${ }^{2,3}$ The realization of $\mathrm{THz} \mu \mathrm{VEDs}$ involves significant issues caused by downward scaling of feature dimension, including electromagnetic wave loss, resonant frequency shift, thermal dissipation, and electron beam transmission. ${ }^{4}$ In addition, the control of surface roughness below skin depth becomes a critical requirement, due to the intrinsically high level of loss at $\mathrm{THz}$ frequencies. This also depends on material conductivity, defects, and the size of grain boundaries. ${ }^{3,5}$

Recent advances in nano- or microfabrication are providing diverse opportunities to exploit frequency operation over $100 \mathrm{GHz}$. These advances include deep-reactive-ion etching (DRIE), X-ray or UV LIGA (German acronym for Lithographie, Galvanoformung Abformung i.e., Lithography, Electroplating, and Molding), polymer micromolding, and growth of diamond in silicon molds. ${ }^{4,6}$ Paoloni et al. presented a deep X-ray photolithography process for 1$\mathrm{THz}$, cascade, backward-wave amplifiers, showing a lateral surface roughness less than $200 \mathrm{~nm}$ and a thickness homogeneity within $3 \mu \mathrm{m} .^{7}$ In the case of DRIE on silicon wafers, a $0.65 \mathrm{THz}$ folded-waveguide (FWG) slow-wave structure was demonstrated by using a two-level, etch process for two

\footnotetext{
a) Author to whom correspondence should be addressed. Electronic mail: cw.baik@samsung.com

b) Current address: Department of Engineering Science, University of Oxford, Oxford OX1 3PJ, U.K.
}

different heights of a FWG trench and a beam tunnel. ${ }^{8,9}$ The etched trenches were then coated with a metal film in thickness of a few multiples of RF skin depth. They also reported a waveguide aspect ratio of exceeding 8:1, a sidewall roughness of $50 \mathrm{~nm}$, and an alignment accuracy of $2 \mu \mathrm{m}$ between two FWG circuit halves. On the other hand, we recently compared RF return loss between measurement and simulation for $0.1 \mathrm{THz}$ backward-wave oscillations, proposing a two-level, ultra-deep $(>200 \mu \mathrm{m})$, reactive ion etching (u-DRIE) process for two different levels of trenches: a beam tunnel half of $280 \mu \mathrm{m}$ and a circuit half of $800 \mu \mathrm{m}$ with an aspect ratio of $8: 1 .^{10,11}$ The measured $13.8 \sim 70.6-\mathrm{nm}$ (rms) surface roughness was well below the skin depth of the surface metal layer, $\mathrm{Au}[\delta(\omega)=\sqrt{2 / \omega \mu \sigma} \sim 240 \mathrm{~nm}]$. However, the suppression of the two-level, u-DRIE process at the narrow channel of resonant cavities changed the aspect ratio and led to growth of vertical microridges on the sidewall of cavities. As a result, not only was a significant RF attenuation observed but also the cutoff and the resonances shifted away from the desired frequencies. ${ }^{11}$

Here, we present a highly precise, three-dimensional (3D), microstructure for $0.1 \mathrm{THz}$ backward-wave interaction circuits fabricated by a multi-level, tandem process. In contrast to the previous works, ${ }^{11,12}$ the interaction circuit is divided into multiple levels in $z-x$ plane along the beam tunnel direction. A cutaway half of the slow-wave circuit is illustrated in Fig. 1. Each level is a separately prepared, 6-in., highly doped, silicon wafer (resistivity $<0.005 \Omega \cdot \mathrm{cm}$ ). The low aspect ratio configuration by splitting into multi levels reduces a plasma-loading effect during DRIE process and provides flat-etched bottom and smooth sidewall profiles. The loading effect is an etch rate variation phenomenon due to difference in pattern density, i.e., the area of exposed silicon, which is unavoidable in complicated feature designs. ${ }^{13}$ Thus, we propose a multi-level fabrication process allowing etch rate uniformity for trenches on each wafer. It is expected to improve both RF attenuation and frequency shifts by reducing structural deviation, enhancing 


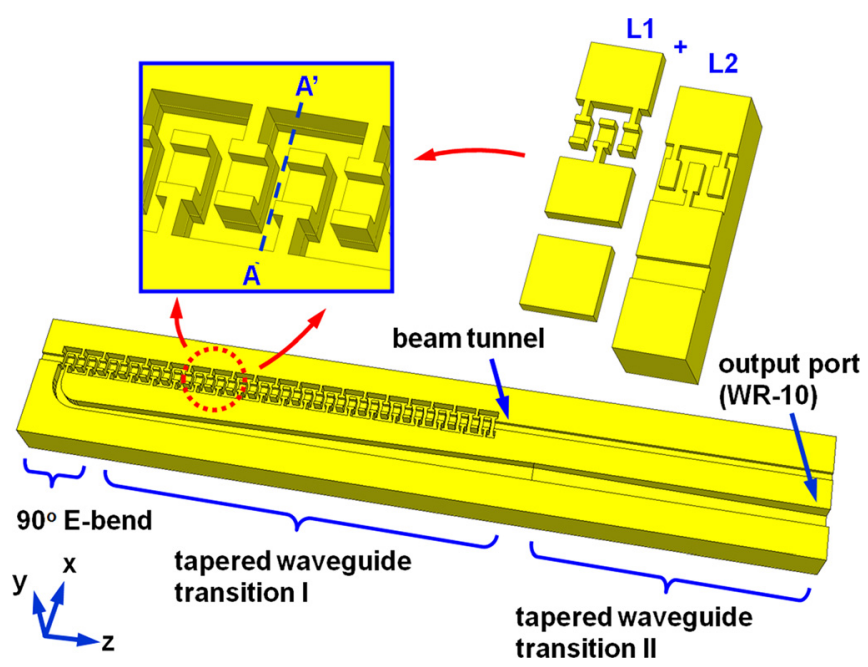

FIG. 1. Perspective view of the $0.1 \mathrm{THz}$ interaction circuit cut in half along the $z-x$ plane.

sidewall roughness, and depositing a conformal metal layer. By measuring scattering parameters of the one-port network, we show that the multi-level, slow-wave circuit provides strong resonances above the cutoff with negligible phase shifts. Then we retrieve an accurate dispersion relation as compared with theoretical predictions. These results may lead to the realization of sophisticated slow-wave microstructures in the $\mathrm{THz}$ frequency regime.

As illustrated in Fig. 2, scanning electron micrograph (SEM) images show the cutaways of the two-wafer, bonded $(L 1+L 2)$ and the four-wafer, bonded $\left(L 2^{\prime}+L 1^{\prime}+L 1+L 2\right)$ circuits. The tandem microstructure consists of the electron beam tunnel with a portion of resonant cavities $\left(L 1, L 1^{\prime}\right)$ and remaining parts $\left(L 2, L 2^{\prime}\right)$. The unit cell of the slow-wave circuit is depicted in Fig. 2(a) along the RF path, $p+h$. We employ a double-sided, u-DRIE process for the top substrate $L 1$, which provides half of beam tunnel $d_{11}$ from the top surface and excavation of cavity trenches $d_{11}+d_{12}$ from both top and bottom surfaces. These are indicated by the arrows in Fig. 2(b). Thus, the top substrate $L 1$ of predetermined thickness $550 \mu \mathrm{m}$ supports through-etched holes for the resonant cavities. This corresponds to the $A-A^{\prime}$ cross section in the inset of Fig. 1. A more detailed process flow and the images of the fabricated circuit are presented in supplementary Figs. S1 and S2. ${ }^{14}$ The aspect ratio (depth:width) in the top substrate $L 1$ for the double-sided, u-DRIE process is less than 1.4:1 $\left(d_{11}=280 \mu \mathrm{m}\right.$ versus the interaction gap, $w_{g}=200$ or $300 \mu \mathrm{m})$. The aspect ratio in the bottom substrate $L 2$ is similarly less than 1.3:1 $\left(d_{2}=260 \mu \mathrm{m}\right.$ versus $w_{g}=200$ or $300 \mu \mathrm{m})$. Two different $w_{g}$ values are chosen to compare return loss characteristics (see supplementary Fig. S3 ${ }^{14}$ ). The etched bottom $d_{2}$ in $L 2$ achieves the height $\Delta$ less than $16.8 \mu \mathrm{m}$ on the bottom of narrow channels by alleviating the plasma-loading effect using low aspect ratios. This is a substantial improvement when compared to $\Delta=80 \mu \mathrm{m}$ in the previous report ${ }^{11}$ where the two-level, u-DRIE process was proposed for two different, high aspect ratio, trench levels.

In particular, we developed an effective method for an etch stop layer when through-etched holes are required in substrate $L 1$. Silicon dioxide or aluminum is commonly used to stop the DRIE process, preventing the leakage of helium
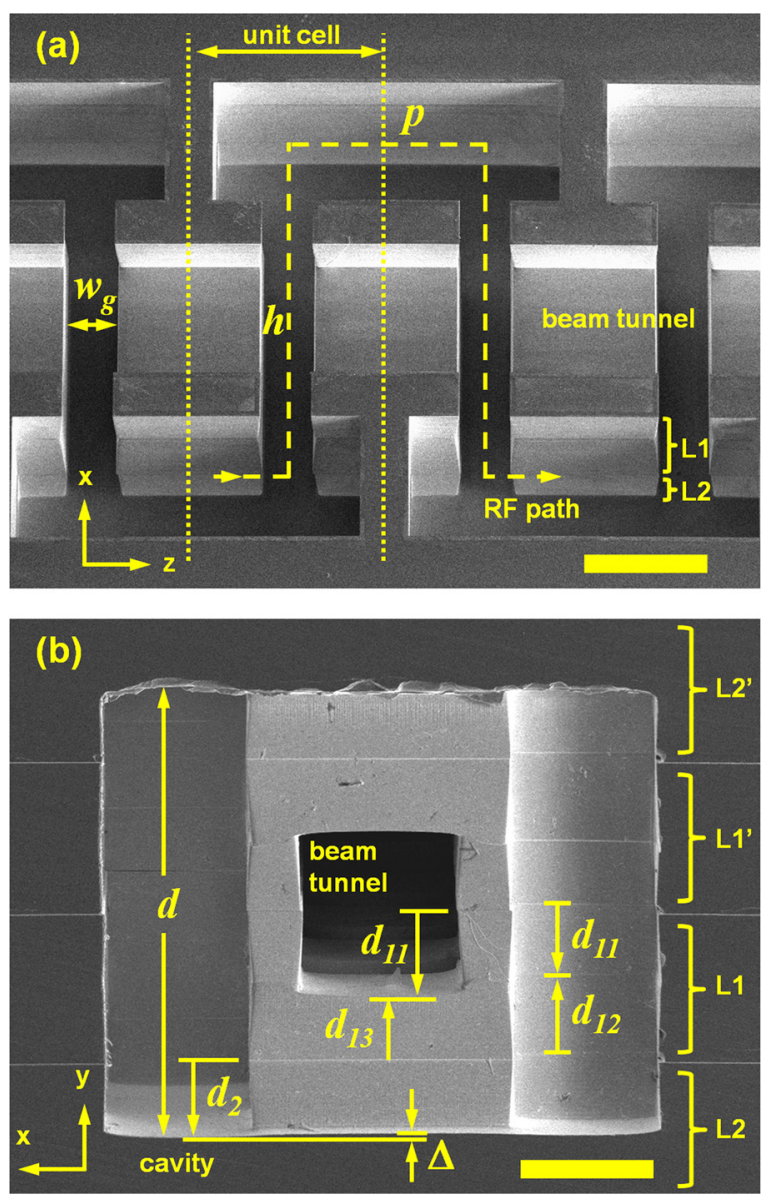

FIG. 2. (a) SEM image of the fabricated interaction circuit after u-DRIE and TCB processes, as conceptually shown in the inset of Fig. 1. Scale bar, $500 \mu \mathrm{m}$. (b) SEM image along the $A-A^{\prime}$ cross section as depicted in Fig. 1 showing the beam tunnel in the center and the multi-level microstructure after TCB process. Scale bar, $500 \mu \mathrm{m}$.

coolant through etched holes, but it may leave a notching on the bottom. ${ }^{13}$ We chose a chemical vapor deposition (CVD) of polymer, Parylene type $\mathrm{C}$, as an etch stop layer on the topside etched trenches $d_{11}$ for conformal and pinhole-free deposition. ${ }^{15}$ Then the backside etch of the substrate $L 1$ provides through-etched holes $d_{11}+d_{12}$ for resonant cavities and waveguide transitions, while the substructure for the beam tunnel $d_{13}$ is protected by masking patterns. The remaining thin membrane of Parylene is removed by oxygen plasmas. A metallic film is deposited on each substrate surface by e-beam evaporation with substrate rotation to obtain conformal, thick, metal layers (500-nm Ti and 1 500-nm Au) with enhanced coverage on the sidewalls. The top $L 1$ and bottom $L 2$ substrates are then engaged together through multiple seal lines by the thermo-compressive bonding (TCB) process described in the previous report. ${ }^{11}$ Finally, the two identical sets of bonded substrates, $L 2^{\prime}+L 1^{\prime}$ and $L 1+L 2$, are faced each other and bonded again with an alignment accuracy of $\pm 2 \mu \mathrm{m}$. Consequently, the configuration of the interaction circuit consists of four, individual levels by single and double-sided, u-DRIE processes on the outer $\left(L 2, L 2^{\prime}\right)$ and inner substrates $\left(L 1, L 1^{\prime}\right)$, respectively.

Figure 3(a) shows the return loss $S_{11}$ of the interaction circuit. The vector network analyzer (HP85107A) measurement is in excellent agreement with two, different, 3D, electromagnetic simulations: finite element method (FEM) and finite 

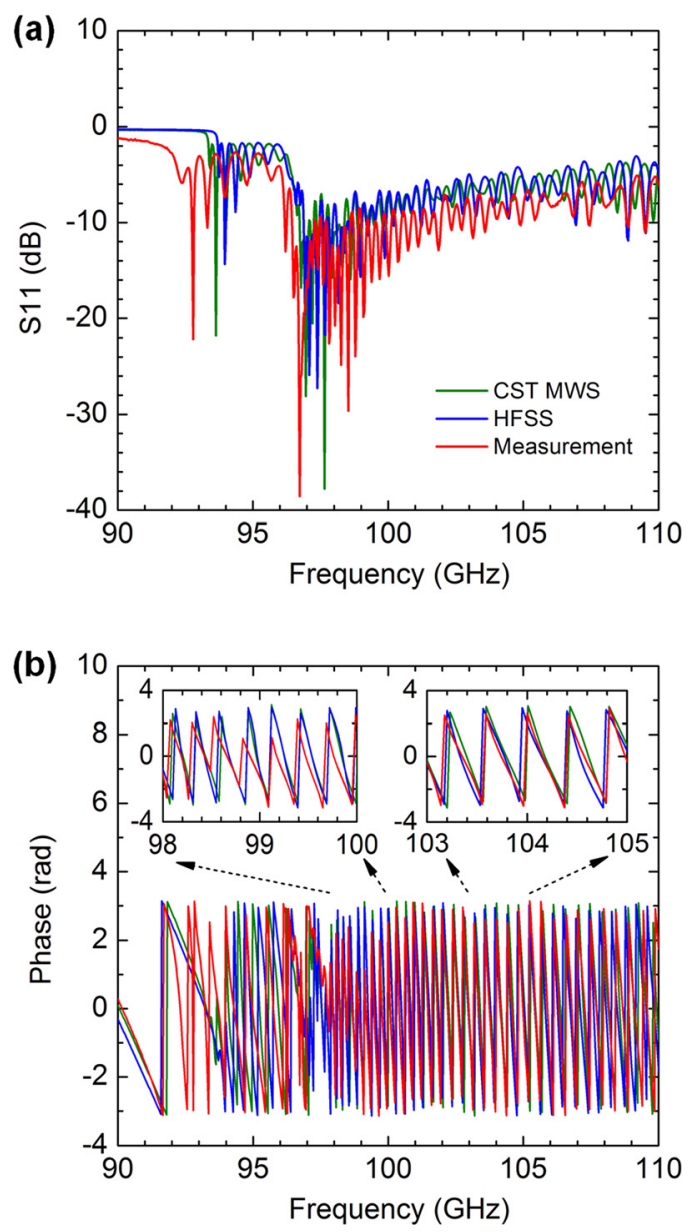

FIG. 3. Simulated and measured return loss characteristics of the interaction circuit: (a) magnitude and (b) phase of $S_{11}$, respectively.

integration technique (FIT), using the Ansoft HFSS Driven Modal Solution ${ }^{16}$ and the CST MICROWAVE STUDIO Transient Solver, ${ }^{17}$ respectively (see supplementary material for the details on measurement and simulation ${ }^{14}$ ). No significant difference was observed in either cutoff or resonant frequencies. A few resonances near $93 \mathrm{GHz}$ are far from cavity resonances, because the circuit resonant frequencies range from 96 to $110 \mathrm{GHz}$, while the cutoff frequency $f_{c}=c / 2 d$ is up to $93.75 \mathrm{GHz}$ at the interface between the tapered transitions I and II (see supplementary Fig. S $7^{14}$ ). Strong resonances appear above the cutoff with no distinctive frequency shift in the region of $98 \sim 110 \mathrm{GHz}$. Furthermore, the multi-level, tandem microstructure for the slow-wave circuit provides substantial improvement in reducing RF attenuation compared to the previous report. ${ }^{11}$ As presented in Fig. 2, we obtain high quality trenches without micro- or nanoscallops, vertical microridges or striations, etch residues, etc. ${ }^{13,18}$ Sidewall scallops are about 10 -nm (rms) surface roughness measured by atomic force microscopy (AFM). Such smooth and clean surface profiles are achievable not only because of low aspect ratios by splitting into multiple levels for u-DRIE process, but also because of the triple, surface smoothing treatment using thermal oxidation and oxide removal. ${ }^{11}$ In addition, the observation of $S_{11}$ phase difference is well consistent with the calculation results, as depicted in Fig. 3(b). In the low frequency region below 98 $\mathrm{GHz}$, the phase mismatch can be attributed to multiple reflections at the $90^{\circ}$ E-bend waveguide and the waveguide transitions (see also supplementary Figs. S6 and $\mathrm{S} 7^{14}$ ). It has to be noted, however, that the phase match between measurement and simulations above $98 \mathrm{GHz}$ appears to be remarkable, as shown in the magnified insets. Less than $0.09 \%$ phase mismatch is observed between measurement and simulation from 98 to $105 \mathrm{GHz}$. Therefore, it may be appropriate to rigorously revisit the characteristics of slow-wave dispersion by the comparison with analytical and numerical calculations. ${ }^{19}$

The previous analytical theory for FWGs ${ }^{20,21}$ is applied here to obtain the dispersion along with the RF path $l_{c}=h+p$ in the cavity. The phase shift is given by $k_{m} p=k_{0}(h+p)+(2 m+1) \pi$, where $k_{m}$ is the propagation constant of the $m$ th space harmonic along the beam tunnel, $k_{0}$ is the fundamental propagation constant along the waveguide, $h$ and $p$ are the height and the period in $x$ and $z$ directions, respectively. Thus, the dispersion relation of the $m$ th space harmonic can be written as $\omega^{2}=\omega_{c}^{2}+k_{0}^{2} c^{2}=$ $\omega_{c}^{2}+c^{2}\left(k_{m} p-(2 m+1) \pi\right)^{2} /(h+p)^{2}$ in terms of the phase shift $k_{m} p$, where $\omega_{c}$ is the cutoff angular frequency of the circuit. The results of dispersion calculation are shown in Fig. 4 with respect to the axial phase shift in the negative group velocity region, $2 \pi<k_{m} p<3 \pi$. The intersection of the beam line with the space harmonic $(m=1)$ generates a coherent backward-wave radiation near $100 \mathrm{GHz}$ with a negative group velocity $\left(v_{g}=d \omega / d\left(k_{m} p\right)<0\right)$. The resonant frequencies are extracted from the return loss measurement and the HFSS Driven Modal Solution, noting the fact that the circuit of $N$ cavities gives $N$ resonances in the fundamental mode with an axial phase shift, $k_{m} p=n \pi / N+2 \pi .^{22}$ The dispersion obtained from the measurement is in good accordance with the simulation result. Here, a few dots near $k_{m} p=2 \pi$ are missing, because they are out of range of the W-band $(75 \sim 110 \mathrm{GHz})$ vector network analyzer. A more detailed description on the dispersion retrieval is given in supplementary material with Fig. S6-Fig. S9 and Table SII. ${ }^{14}$ The measured and simulated wave dispersions show, however, a frequency deviation from the analytical

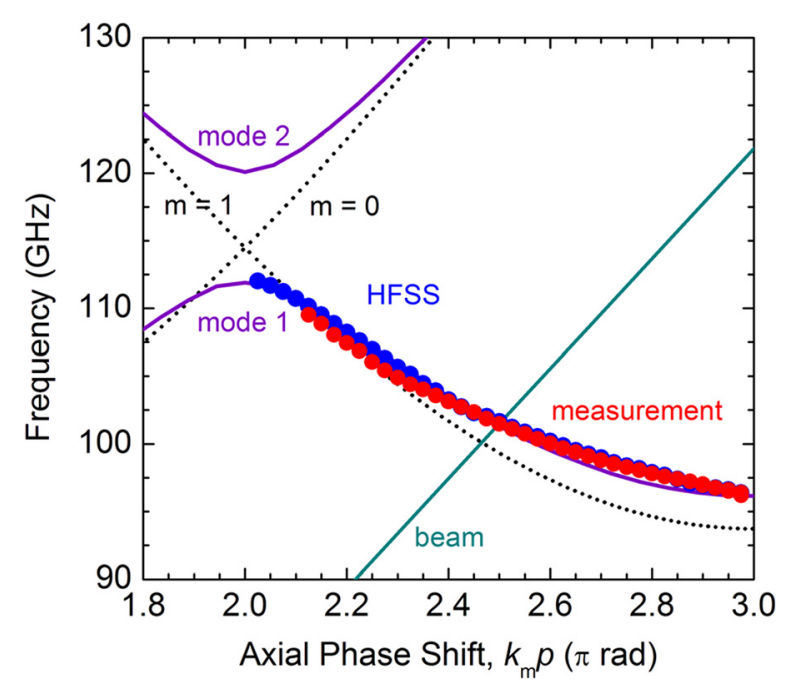

FIG. 4. Dispersion relations from the analytical calculation (black dotted lines) and the HFSS Eigenmode Solution for the single unit cell (violet-colored, solid lines). Dispersion of the electron beam with $12 \mathrm{kV}$ is plotted in a cyan-colored, solid line. Retrieved dispersions from the return loss measurement and the HFSS Driven Modal Solution are given in red and blue dots, respectively, for the full circuit geometry as illustrated in Fig. 1. 
prediction along with the axial phase shift. This discrepancy reaches up to $2.5 \mathrm{GHz}$ at $k_{m} p=3 \pi$, where the frequency is close to the cutoff. It may be attributed to the fact that the fringing fields on both sides of the rectangular aperture in the beam tunnel contribute to the increase of discontinuity reactance in wave propagation. ${ }^{23,24}$ Such an effect is not considered in the analytical calculation (see supplementary Fig. $\mathrm{S} 10^{14}$ ). We also carried out the HFSS Eigenmode Solution ${ }^{16}$ for the single unit cell structure, which provides an efficient way in analyzing periodic slow-wave circuits. Then, we found the lowest passband (mode 1), i.e., the cavity mode ${ }^{22}$ and the next higher passband (mode 2), called the slot mode near the upper cutoff of the lowest passband. The cavity mode obtained from the HFSS Eigenmode Solution corresponds well to the measurement. The accuracy of the HFSS Eigenmode Solution is discussed in supplementary Table SIII. ${ }^{14} \mathrm{We}$ consequently retrieved the highly accurate dispersion relation from the multi-level, slow-wave structure as compared with computational simulations.

In summary, the proposed multi-level, tandem microstructure for slow-wave circuits demonstrates excellent agreement with theoretical prediction of return loss characteristics. It is noteworthy that splitting into multiple levels enables a more sophisticated approach for resonant frequencies and phases, together with RF attenuation. The wave dispersion relation then leads to a clear fingerprint for experimentally identifying and characterizing interaction circuits. We therefore conclude that the multilevel, tandem configuration for slow-wave circuits provides precise realization of $\mathrm{RF}$ characteristics. This would be useful for the development of future $\mathrm{THz}$ $\mu$ VEDs.

The authors are grateful to Dr. Seog-Tae Han at Korea Astronomy \& Space Science Institute, Suren Singh at Agilent Technologies, Inc., Aaron Jensen at SLAC National Accelerator Laboratory, David Vondran and Yuenie Lau at
OML, Inc. for their technical assistance, particularly in measuring S-parameters using W-band vector network analyzers.

${ }^{1}$ M. Tonouchi, Nature Photon. 1, 97 (2007).

${ }^{2}$ J. H. Booske, Phys. Plasmas 15, 055502 (2008).

${ }^{3}$ Y. M. Shin, L. R. Barnett, D. Gamzina, N. C. Luhmann, Jr., M. Field, and R. Borwick, Appl. Phys. Lett. 95, 181505 (2009).

${ }^{4}$ J. H. Booske, R. J. Dobbs, C. D. Joye, C. L. Kory, G. R. Neil, G. S. Park, J. Park, and R. J. Temkin, IEEE Trans. Terahertz Sci. Technol. 1, 54 (2011).

${ }^{5}$ N. Laman and D. Grischkowsky, Appl. Phys. Lett. 93, 051105 (2008).

${ }^{6}$ R. L. Ives, IEEE Trans. Plasma Sci. 32, 1277 (2004).

${ }^{7}$ C. Paoloni, A. D. Carlo, F. Bouamrane, T. Bouvet, A. J. Durand, M. Kotiranta, V. Krozer, S. Megtert, M. Mineo, and V. Zhurbenko, IEEE Trans. Electron Dev. 60, 1236 (2013).

${ }^{8}$ K. E. Kreischer, J. C. Tucek, D. A. Gallagher, and R. E. Mihailovich, in Proceedings of 33rd International Conference on Infrared, Millimeter, and Terahertz Waves, Pasadena CA, 2008.

${ }^{9}$ J. C. Tucek, M. Basten, D. Gallagher, and K. E. Kreischer, in Proceedings of IEEE International Vacuum Electronics Conference (IVEC), 2010.

${ }^{10}$ C. W. Baik, S. Hong, and H. Suh, U.S. patent 8,188,799 (29 May 2012).

${ }^{11}$ C. W. Baik, S. Hong, J. Kim, H. Y. Ahn, J. H. Choi, S. I. Kim, J. Lee, Y. Kim, J. M. Kim, S. Hwang, S. Y. Jun, and S. Yu, Appl. Phys. Lett. 101, 073508 (2012).

${ }^{12}$ Y. M. Shin, A. Baig, R. Barchfeld, D. Gamzina, and L. R. Barnett, Appl. Phys. Lett. 100, 154103 (2012).

${ }^{13}$ B. Wu, A. Kumar, and S. Pamarthy, J. Appl. Phys. 108, 051101 (2010).

${ }^{14}$ See supplementary material at http://dx.doi.org/10.1063/1.4862324 for additional Figures.

${ }^{15}$ K. R. Williams, K. Gupta, and M. Wasilik, J. Microelectromech. Syst. 12, 761 (2003).

${ }^{16}$ High Frequency Structure Simulator (HFSS) version 13.0, Ansys, Inc., Canonsburg, PA, 2013.

${ }^{17}$ CST Studio Suit (Microwave Studio), version 2009.

${ }^{18}$ C. K. Kang, S. M. Lee, I. D. Jung, P. G. Jung, S. J. Hwang, and J. S. Ko, J. Micromech. Microeng. 18, 075007 (2008).

${ }^{19}$ K. C. Zhang, Z. H. Wu, and S. G. Liu, Chin. Phys. B 17, 3402 (2008).

${ }^{20}$ H. J. Ha, S. S. Jung, and G. S. Park, J. Korean Phys. Soc. 34, 297 (1999).

${ }^{21}$ S. T. Han, K. H. Jang, J. K. So, J. I. Kim, Y. M. Shin, N. M. Ryskin, S. S. Chang, and G. S. Park, IEEE Trans. Plasma Sci. 32, 60 (2004).

${ }^{22}$ F. Kantrowitz and I. Tammaru, IEEE Trans. Electron Dev. 35, 2018 (1988).

${ }^{23}$ J. H. Booske, M. C. Converse, C. L. Kory, C. T. Chevalier, D. A. Gallagher, K. E. Kreischer, V. O. Heinen, and S. Bhattacharjee, IEEE Trans. Electron Dev. 52, 685 (2005).

${ }^{24}$ M. Sumathy, K. J. Vinoy, and S. K. Datta, IEEE Trans. Electron Dev. 57, 1440 (2010). 